\section{Gesundheitsziele für Deutschland: Eine Initiative wird gestartet}

Ute Brasseit, Matthias Wismar, Sybille Angele, Anke Bramesfeld

Medizinische Hochschule Hannover

psychoneuro 2003; $29(6):$ 296-298

Gesundheitsziele als Instrument zur inhaltichen Ausrichtung und Fokussierung von Gesundheitspolitik setzen sich in den letzten Jahren zunehmend durch. Zahlreiche OECD Staaten verfügen über eigene Gesundheitszielprogramme so z.B. Großbritannien, Australien oder die USA. Die Anzahl der Gesundheitszielprogramme auf regionaler Ebene ist kaum mehr zu überblicken. Der folgende Artikel beschreibt den Prozess und die theoretischen Hintergründe, die zur Auswahl der spezifischen Gesundheitszielthemen des deutschen nationalen Gesundheitszielprogramms geführt haben.

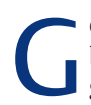
esundheitsziele sind vereinbarte Ziele, die in einem festgelegten Zeitraum zu erreichen sind. Sie können direkt auf die Verbesserung der Gesundheit in definierten Bereichen oder für bestimmte Gruppen ausgerichtet sein, aber auch auf verbesserte Strukturen, die Einfluss auf die Gesundheit der Bevölkerung und die Krankenversorgung haben. Gesundheitsziele dienen der Priorisierung von besonderen Gesundheitsproblemen und -risiken und gesundheitsbezogenen Belangen der Bürger(inne)n und Patient(inn)en. Gesundheitsziele sollen

- durch die Priorisierung den politischen Diskurs über die Weiterentwicklung von Gesundheitsförderung, Prävention, Kuration und Rehabilitation vorantreiben

- die für Gesundheit zur Verfügung stehenden finanziellen Mittel problemangemessen lenken und

- neue Impulse für Management und Leistungserbringung vor Ort setzen.

Während sich der Gesundheitszielgedanke schon seit langem in den Bundesländern etabliert hat, existiert auf Bundesebene seit dem Jahr 2000 unter dem Titel gesundheitsziele.de eine Initiative der Akteure im Gesundheitswesen zur Erarbeitung von Gesundheitszielen. Die Initiative steht unter der Schirmherrschaft der Gesellschaft für Versicherungswissenschaft und -gestaltung (GVG e.V.) in Kooperation mit dem Bundesministerium für $\mathrm{Ge}$ sundheit und soziale Sicherung (BMGS) und vereinigt zahlreiche Akteure des Gesundheitswesens in sich. Am 14. Februar 2003 wurde der Bericht über die geplanten konkreten Ziele und Maßnahmen des deutschen Gesundheitszielprogramms der Bundesministerin für Gesundheit und Soziale Sicherung übergeben (einsehbar unter www.gesundheitsziele.de).

\section{Auswahl von Zielbereichen und Themen}

Der Auswahl von Zielbereichen und von Themen für ein nationales Gesundheitsziel-Programm scheinen auf den ersten Blick keine Grenzen gesetzt zu sein. Würden $\mathrm{Ge}-$ sundheitsziele jedoch nur unter dem Gesichtspunkt „Reduzierung der Sterblichkeit“ oder „Gewinn an Lebensjahren“ ausgewählt, würden Themen wie psychische Erkrankungen $(5,12)$ oder Allergien, die einen erheblichen Einfluss auf die Lebensqualität der Betroffenen haben können, systematisch unterrepräsentiert. Würden Gesundheitsziele nur auf Krankheitsbilder abzielen, ergäbe sich aller Wahrscheinlichkeit nach eine einseitige Ausrichtung auf kurative Ansätze und das Ergebnis wären reine „Versorgungsziele“, bei welchen Aspekte der Prävention und Gesundheitsförderung weitgehend vernachlässigt würden. gesundheitsziele.de entschied sich daher, das Finden möglicher Gesundheitszielthemen aus den Perspektiven unterschiedlicher Zielbereiche heraus anzugehen (Abb. 1). Die Zielbereiche weisen dabei ausdrücklich keine Hierarchisierung auf, sondern werden gleichwertig betrachtet. Diese Zielbereiche zeichnet eine ausgesprochene Public-Health- bzw. Gesundheitssystemperspektive aus, wie sie auch im Ansatz der WHO für Gesundheitsziele verwirklicht ist $(14,15)$. Diese Perspektive ist mit einer Vielzahl von Vorteilen verbunden:

- Gesundheitlich relevante Gesellschaftsbereiche können so über das Gesundheitswesen im engeren Sinne als Interventionsbereiche ins Auge gefasst werden. Dies ist z.B. bei der Wahl eines Setting-Ansatzes für Präventionsmaßnamen für bestimmte Bevölkerungsgruppen, wie z.B. 
Kinder in Kindertagesstätten/ Schulen $(3,6)$ oder sozial Benachteiligte in der Kommune, sinnvoll (7-9,11)

- Rein krankheits-, risikofaktorenoder verhaltensorientierte Ansätze können so durch verhältnisorientierte Ansätze sinnvoll ergänzt werden

- Schließlich trägt eine solche Vorgehensweise dem Wandel des Krankheitsspektrums hin zu chronischen Erkrankungen - unter denen die psychischen Erkrankungen von wachsender Bedeutung sind (5) - Rechnung. Die im Zusammenhang mit diesem veränderten Krankheitsspektrum große Anzahl zuständiger Träger und Akteure in den verschiedenen Sektoren lässt sich entlang des Krankheitsbzw. Versorgungskontinuums einfacher beteiligen und koordinieren (12).

\section{Definition von Zielen}

Die Auswahl von Gesundheitszielen wirft fast unweigerlich die Frage nach der Priorisierung von Gesundheitsthemen auf. gesundheitsziele.de will jedoch nicht Gesundheitsthemen zum Nachteil anderer vorrangig bearbeiten. Einige wenige Gesundheitsziele sollen beispielhaft entwickelt werden, um zu untersuchen, ob sich Ziele verschiedenen Charakters in Deutschland zusammen mit relevanten Akteuren und Betroffenen realisieren lassen. Da jedoch auch eine beispielhafte Auswahl transparent und nachvollziehbar gestaltet werden muss, wurden für die Auswahl von Themen Auswahlkriterien (Abb. 2) konzipiert. Um dafür eine annähernd wissenschaftliche Basis zu gewinnen und trotzdem pragmatisch - im Sinne von handlungsorientiert - vorzugehen, wurde in einem Vergleich auf vorhandene supranationale, nationale und regionale Gesundheitszielprogramme und Priorisierungs-Initiativen zurückgegriffen.

\section{So wissenschaftlich wie nötig, so} praxisnah wie möglich

Um eine umfassende Übersicht möglicher Gesundheitsziel-Themen für den Auswahlprozess bei gesundheitsziele.de $\mathrm{zu}$ gewinnen, wurden u.a. die Zielthemen des WHO-Ansatzes „Health for All“ und die Zielprogramme aus Australien, Großbritannien und einigen deutschen Bundesländern herangezogen (2) und so eine Art „Zielekorb“ erstellt. Dieser Vorgehensweise liegt die Annahme zugrunde, dass Erfahrungen aus Ländern mit vergleichbarem Entwicklungs- und Wohlstandsniveau und damit ähnlichem Krankheitsspektrum auf Deutschland übertragbar seien. Die weiteren Schritte bestanden darin, die möglichen Themen aus dem so gewonnen „Zielekorb“ mit wissenschaftlich fundierten Informationen zur Einschätzung der Bedeutung verschiedener Gesundheitsthemen abzugleichen.
Hierzu wurden z.B. die Studie „Global Burden of Disease“ (4,5), die Ergebnisse der „Projektgruppe Prioritäre Gesundheitsziele“ in Deutschland (13), die Ergebnisse des Leitlinien-Clearing-Verfahrens in Deutschland (1) und der Gesundheitsbericht für Deutschland (10) herangezogen. Dabei wurde u.a. die Relevanz des Themas „Depression“ deutlich, was zur zusätzlichen Aufnahme des Themas führte. Anschließend wurden die Themen für gesundheitsziele.de nach den oben beschriebenen gemeinsam abgestimmten Kriterien (Abb. 2) in einem ausführlichen und demokratischen Diskussionsprozess im Sommer 2001 von den Mitgliedern der GVG und im Konsens verabschiedet (16).

Als Ergebnis dieses Prozesses wurde sich schließlich auf die in Abbildung 3 dargestellten Gesundheitsziele geeinigt. Die fünf Gesundheitszielthemen, von denen angenommen wurde, dass bundesweit die meisten Erfahrungen in Bezug auf Präventions- und Gesundheitsförderungsprojekte bestünden, sollten zuerst angegangen werden. Die anderen Themen, insbesondere das Thema Depression, sollen im Jahr 2003 nachfolgen.

\section{Abb. 1 Zielbereiche für gesundheitsziele.de}

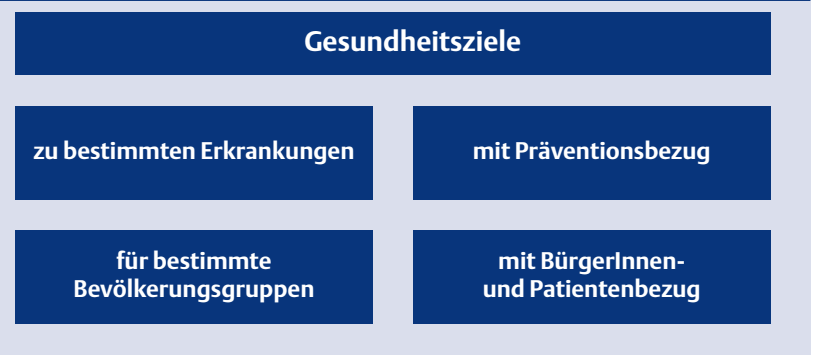

\section{Abb. 2 Von gesundheitsziele.de gewählte Kriterien der Auswahl von Gesundheitsthemen}

Schweregrad des
Gesundheitlichen Problems:
Mortalität
Verbesserungspotenzial
Förderung von Chancengleichheit
Machbarkeit hinsichtlich der
verfügbaren und wirksamen
Instrumente (Maßnahmen)

Schweregerad des

Gesundheitlichen Problems: Individuelle Krankheitslast

Volkswirtschaftliche Relevanz

Priorität des Problems aus Sicht der Bevölkerung

Machbarkeit hinsichtlich der an gesundheitsziele.de beteiligten Akteure
Verbreitung

Ethische Aspekte

Messbarkeit der Ergebnisse

Beteiligungsmöglichkeiten von BürgerInnen und PatientInnen 
Abb. 3 Das Ergebnis des Konsensusprozesses: Gesundheitszielthemen für ein nationales Gesundheitszielprogramm

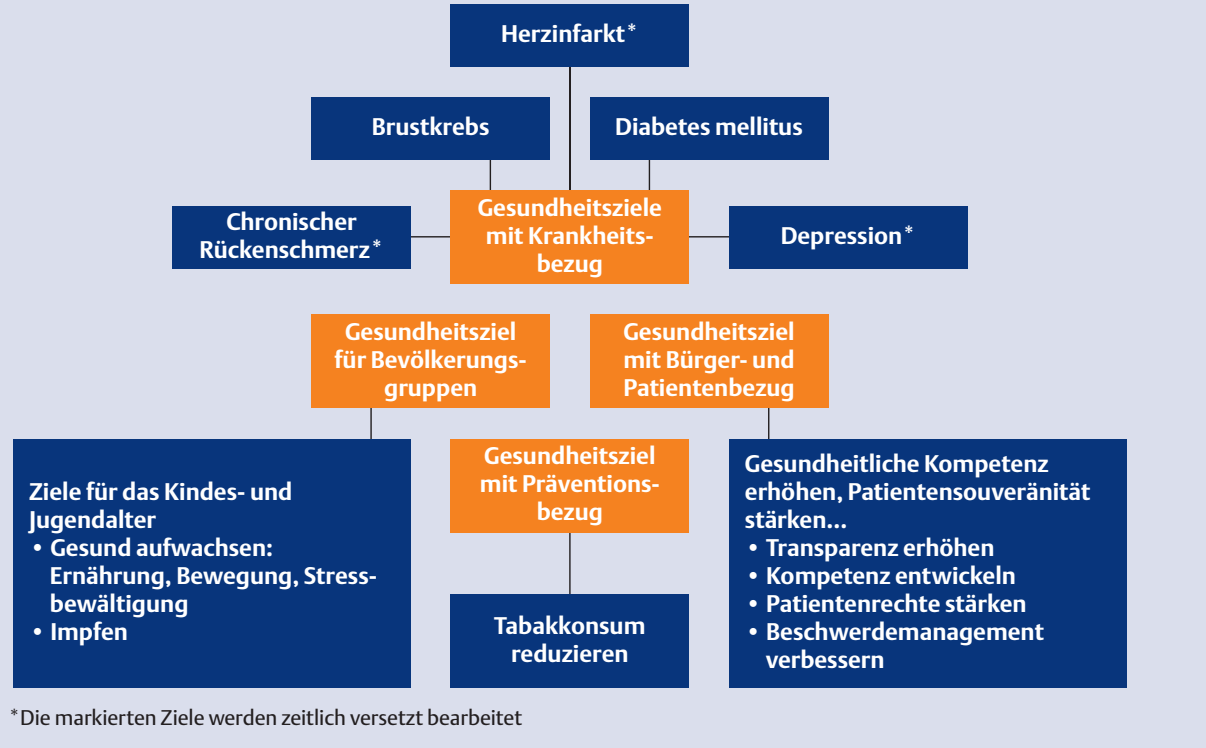

\section{Key Words}

health targets - health policy - priority setting

\section{Literatur \\ 1. ÄZQ - Ärztliche Zentralstelle Qualitäts- sicherung. Priorisierung von Gesundheits- oder Versorgungsproblemen als Themen des Leitlinien-Clearing-Verfahrens. Konzept der AG Priorisierung der Erweiterten Pla- nungsgruppe zur Priorisierung von Leitli- nien-Themen. unveröffentlichtes Manus- kript}

2. Brasseit U, Wismar M. Inhaltliche Ausrichtung und konkrete Themen - Zielkorb zur exemplarischen Auswahl von gesundheitsziele.de. In: GVG - Gesellschaft für Versicherungswissenschaft und -gestaltung (Hrsg.): Gesundheitsziele.de - Forum Gesundheitsziele Deutschland: Gesundheitsziele für Deutschland: Entwicklung, Ausrichtung, Konzepte. Akademische Verlagsgesellschaft Aka GmbH, Berlin, 2002: 71-100 3. Hurrelmann K. Gesundheitssituation von Kindern und Jugendlichen: Konsequenzen für die Gesundheitsförderung. Die BKK, Schwerpunktthema Kinder und Jugendliche 2001; 9: 424

4. Murray CJL, Lopez AD (Hrsg.). The Global Burden of Disease. A comprehensive assessment of mortality and disability from diseases, injuries, and risk factors in 1990 and projected to 2020. Cambridge, Harvard School of Public Health (distributed by Harvard University Press), on behalf of the World Health Organization and the World Bank, 1996

5. Murray CJL, Lopez AD. The Global Burden of Disease and Injury: Executive Summary. 1996
6. Paulus P. Gesundheitsfördernde Schulen als Gegenpotenzial zum Leben? In: Altgeld T, Hofrichter P (Hrsg.). Reiches Land -kranke Kinder? Gesundheitliche Folgen von Armut bei Kindern und Jugendlichen. Frankfurt am Main, Mabuse-Verlag, 2000: 235-259

7. Rosenbrock R. Primärprävention zur Verminderung sozial bedingter Unterschiede von Gesundheitschancen - was ist das und welche Rolle können die Krankenkassen dabei spielen? Arbeit und Sozialpolitik, 2001; 11-12: 49-57

8. Siegrist J, Joksimovic L. „Tackling Inequalities in Health“ - ein Projekt des „European Network of Health Promotion Agencies" (ENHPA) zur Gesundheitsförderung bei sozial Benachteiligten. Abschlussbericht für das deutsche Teilprojekt. Düsseldorf, Heinrich Heine Universität Düsseldorf, Medizinische Einrichtungen, Institut für Medizinische Soziologie, 2000

9. Spitzenverbände der Krankenkassen. Gemeinsame und einheitliche Handlungsfelder und Kriterien der Spitzenverbände der Krankenkassen zur Umsetzung von § 20 Abs. 1 und 2 SGB V vom 21. Juni 2000 in der Fassung vom 27. Juni 2001. unveröffentlichtes Manuskript

10. StBA - Statistisches Bundesamt (Hrsg..: Gesundheitsbericht für Deutschland. Wiesbaden, Metzler-Poeschel, 1998

11. Stuppardt R. Von der kurativen zur präventiven Medizin. Neuausrichtung der Gesundheitssicherung. Soziale Sicherheit, 2001; 8-9: 1-7

12. SVR Sachverständigenrat für die Konzertierte Aktion im Gesundheitswesen: Bedarfsgerechtigkeit und Wirtschaftlichkeit. Gutachten 2000/2001. Band 3. Über-, Un- ter- und Fehlversorgung. Ausführliche Zusammenfassung. Baden-Baden, Nomos Verlagsgesellschaft, 2001

13. Weber I, Abel M, Altenhofen L, Bächer K, Berghof B, Bergmann KE, Flatten G, Micheelis W, Müller PJ. Dringliche Gesundheitsprobleme der Bevölkerung in der Bundesrepublik Deutschland: Zahlen - Fakten - Perspektiven. Baden-Baden, Nomos Verlagsgesellschaft, 1990

14. WHO Regional Office for Europe: Ziele zur „Gesundheit für alle“. Die Gesundheitspolitik für Europa. Weltgesundheitsorganisation Regionalbüro für Europa, Kopenhagen, 1993

15. WHO Regional Office for Europe: Health21 - The Health for All Policy for the WHO European Region - 21 Targets for the 21st Century. Copenhagen, 1998

16. Wismar M, Brasseit U, Ollenschläger $G$, Angele S. Verfahren und Kriterien zur exemplarischen Auswahl von Gesundheitszielen. In: GVG - Gesellschaft für Versicherungswissenschaft und -gestaltung (Hrsg.): Gesundheitsziele.de - Forum Gesundheitsziele Deutschland: Gesundheitsziele für Deutschland: Entwicklung, Ausrichtung, Konzepte. Berlin, Akademische Verlagsgesellschaft Aka GmbH, 2002: 17-38

\section{Korrespondenzadresse:}

Dipl.-Sozialwiss. Ute Brasseit

Medizinische Hochschule Hannover

Abteilung Epidemiologie, Sozialmedizin und Gesundheitssystemforschung OE 5410 30623 Hannover

e-Mail: brasseit.ute@mh-hannover.de 